\title{
Differential growth of brain and retinal bovine pericytes
}

\author{
H. C. Wong ${ }^{1}$, S.M. Elts ${ }^{1}$, J. W. Phillips ${ }^{2}$ and K. A. Williams ${ }^{1}$ \\ ${ }^{1}$ Department of Ophthalmology and ${ }^{2}$ Department of Biochemistry and Chemical Pathology, Flinders Medical Centre, Bedford Park, \\ South Australia, Australia
}

\begin{abstract}
Summary. Within the central nervous system, pericyte degeneration in diabetes mellitus occurs only in the retinal microcirculation and is not seen in the brain. This study sought to elucidate differences between bovine retinal and brain pericytes. When pairs of retinal and brain pericytes from individual calves were cultured in vitro, the morphological organisation of early post-confluent retinal pericyte cultures was consistently different from that of brain pericyte cultures. When retinal and brain pericyte cultures were grown to second passage in high or normal glucose medium supplemented with fetal calf serum, brain pericyte cultures grew significantly faster than retinal pericytes in either medium $(p<0.0001)$. Brain pericytes thus appeared to grow intrinsically faster than retinal pericytes and this effect was largely independent of glucose concentration. Brain pericytes also grew faster than retinal pericytes in high glucose
\end{abstract}

medium containing human diabetic or control serum $(p<0.002)$. The proliferative effect of serum from diabetic patients with non-proliferative diabetic retinopathy on pericytes grown in high glucose medium was not significantly different from that of control serum. Both brain and retinal pericytes showed variation in their ability to replicate in high concentrations of glucose. The selectivity of pericyte degeneration to the retinal circulation does not appear to be due to changes in the mitogenic activity of diabetic serum for retinal pericytes, but may relate to the intrinsic relative inability of the retinal pericyte to reproliferate in response to the metabolic injury of diabetes mellitus.

Key words: Brain, retina, pericytes, glucose, growth rates, diabetic serum.
Since the 1950s, the intramural pericyte has been known to be present in the mammalian cerebral $[1,2]$ and retinal microvasculature [3]. Because the retina is embryologically derived from the fetal forebrain, the biological behaviour of the pericytes in these two locations would be expected to be the same in health and disease. However, in diabetes mellitus this does not appear to be the case.

A hallmark of diabetic retinopathy was shown by Cogan et al. [4] to be the degeneration of the intramural pericyte, which occurs early in the development of retinopathy. Subsequent clinico-pathologic studies by Speiser et al. [5] and by Kohner and Henkind [6] have confirmed that degeneration is present prior to the development of proliferative diabetic retinopathy. Although microvascular pericyte degeneration has been shown to occur in the muscles of diabetic patients [7], within the central nervous system this degenerative process appears to be restricted to the retina [8], an enigma that has been emphasized by Ashton and Tripathi [9] and by Frank [10].

Within the confines of the central nervous system, there has been no satisfactory explanation for the selectiv- ity of pericyte degeneration to the retinal circulation. Although excessive polyol production has been implicated in the pathogenesis of pericyte degeneration, biochemical evidence of aldose reductase activity has been found in bovine microvessels of both brain and retina by Kennedy et al. [11]. Using immunocytochemical techniques, Akagi et al. [12] have shown that aldose reductase is also present in human retinal microvessels, but the absence of this enzyme in human cerebral microvessels, which would account for the selectivity of pericyte degeneration to the retina, has not yet been demonstrated. Furthermore, though excessive polyol producted has been consistently associated with retinal pericyte degeneration in animal models, the use of aldose reductase inhibitors in these models has not always prevented the development of pericyte ghosts $[13,14]$, suggested that polyol production may be a factor associated with pericyte degeneration, but not its sole cause.

In the present study, we have investigated the growth characteristics of pairs of bovine retinal and brain pericytes that were cultured in the presence of fetal calf serum, 
or in human serum from diabetic or non-diabetic patients. Any given retinal and brain pericyte pair was always prepared from the same individual animal. Specifically, we have examined the possibility that during the early phase of diabetes, there may be a systemic factor in the blood in addition to high glucose, that leads to reduced growth of retinal pericytes. In order to seek this putative factor, we have compared the mitogenic activity of non-proliferative diabetic serum for bovine brain and retinal pericytes with that of non-diabetic serum. We chose to use bovine pericytes to allow for preparation of adequate brain and retinal pericytes for the mitogenic assays, and due to the difficulties associated with the acquisition of human material.

\section{Subjects, materials and methods}

\section{Isolation and culture of bovine cells}

\section{All bovine tissues were acquired from a local abattoir.}

Brain and retinal pericytes. The isolation and long-term culture of pericytes from brain and retina of the same calf were performed as previously described [15], with the following modifications. Because the amount of retinal material obtainable from one animal is small, both retinas were gently homogenised by hand using three up/down strokes in a teflon-glass homogeniser. Access to the parietal grey cortex of the calf was gained via a craniotomy using a rotary dental saw. Calves aged less than 6 months were used, since older animals had such large heads and thickened skulls that aseptic access to the cerebrum was very difficult. The meninges were dissected away and a $2 \mathrm{~cm}^{3}$ cube of grey cortex was cut out under aseptic conditions. The cerebral tissue was homogenised using a rotary homogeniser driven at $250 \mathrm{rev} / \mathrm{min}$ for two up/down strokes. Partial digestion of the small amount of retinal tissue was achieved using $10 \mathrm{ml}$ of a combination of enzymes [15] for a period up to $5 \mathrm{~min}$. Since there was a larger mass of brain tissue, digestion lasted for a period of $5 \mathrm{~min}$. The enzymatic process was monitored frequently by light microscopy, to determine the end-point when a majority of pericytes was still adherent to microvessels.

Both brain and retinal pericytes were grown in high glucose-Dulbecco's modified Eagles medium with a glucose concentration of $25 \mathrm{mmol} / 1$ (high glucose-DMEM; Flow Laboratories, North Ryde, New South Wales, Australia), supplemented with $20 \%$ volume/volume (vol/vol) fetal calf serum (Flow Laboratories) to a final glucose concentration of $21.4 \mathrm{mmol} / \mathrm{l}$, on $25 \mathrm{~cm}^{2}$ uncoated culture flasks (Costar Corporation, Cambridge, Mass., USA). Because brain pericytes grew faster than retinal pericytes, the former were held at a post-confluent state until the retinal pericytes had reached post-confluence. At that time, both types of pericytes were passaged. Brain and retinal pericytes were sub-cultured in a split ratio of $1: 3$. Since endothelial celis require a substrate for growth, enriched pericyte cultures were obtained.

Retinal capillary endotinelial cells, aortic endothelial cells and aortic smooth muscle cells. Retinal capillary endothelial cells were isolated and cultured according to the method described by Wong et al. [15]. Aortic endothelial cells were prepared from bovine aorta. The wall of the ascending aorta was cut through longitudinally. The lumen was washed with copious quantities of phosphate-buffered saline (Dulbecco's A: $0.137 \mathrm{~mol} / 1 \mathrm{NaCl}, 2.68 \mathrm{mmol} / \mathrm{l} \mathrm{KCl}, 10.00 \mathrm{mmol} / \mathrm{l}$ $\mathrm{Na}_{2} \mathrm{HPO}_{4}, 1.47 \mathrm{mmol} / \mathrm{K} \mathrm{KH}_{2} \mathrm{PO}_{4} ; \mathrm{pH}=7.4$ ). Endothelium was detached by one very gentle wipe of a scalpel blade. The resultant scroll of endothelium, attached to the blade, was transferred to a $60 \mathrm{~mm}$ culture dish (Costar Corporation) filled with normal glucoseDMEM (glucose concentration $=5.5 \mathrm{mmol} / \mathrm{l}$ ) supplemented with $10 \% \mathrm{vol} / \mathrm{vol}$ fetal calf serum, and inspected by phase-contrast microscopy to ensure that no adventitial cells were attached. The scroll was then diced into fragments with a blade and the pieces allowed to attach to the culture dish. To isolate smooth muscle cells, endothelium was detached from the aorta as described above, exposing the medial smooth muscle layer. A small square of the medial wall was dissected off and transferred to a $60 \mathrm{~mm}$ culture dish (Costar Corporation) filled with normal glucose-DMEM supplemented with $10 \% \mathrm{vol} / \mathrm{vol}$ fetal calf serum. Smooth muscle cells grew readily from the explant. All cells were grown at $37^{\circ} \mathrm{C}$ in an atmosphere of air $/ 5 \%$ $\mathrm{CO}_{2}$ and subcultured at confluence at a split ratio $1: 3$.

\section{Flow cytometry}

Near-confluent pericyte cultures were harvested using $0.025 \%$ weight/volume (wt/vol) trypsin- $0.02 \% \mathrm{wt} / \mathrm{vol}$ EDTA $\left(2 \mathrm{~min}, 37^{\circ} \mathrm{C}\right)$ and the detached cells were immediately washed in cold DMEM with $20 \%$ fetal calf serum to stop the reaction. Viability was assessed by trypan blue exclusion. Cell suspensions were adjusted to $5 \times 10^{6}$ viable cells $/ \mathrm{ml}$ in phosphate-buffered saline containing $0.02 \mathrm{~mol} / \mathrm{l} \mathrm{so-}$ dium azide. Indirect immunofluorescence assays were performed as described by Zola [16]; in brief, $2.5 \times 10^{5}$ cells were incubated with a saturating amount of monoclonal antibody for $30 \mathrm{~min}$ at $4{ }^{\circ} \mathrm{C}$, washed twice in ice-cold phosphate-buffered saline-azide $(5 \mathrm{~min}$, $4^{\circ} \mathrm{C}, 400 \mathrm{~g}$ ), incubated with a fluorescein isothiocyanate-conjugated, affinity-isolated $\mathrm{F}\left(\mathrm{ab} \mathrm{b}^{\prime}\right)_{2}$ fraction of sheep anti-mouse immunoglobulin (catalogue code DDAF; Silenus Laboratories, Hawthorn, Victoria, Australia) for $30 \mathrm{~min}$ at $4^{\circ} \mathrm{C}$, washed two more times in ice-cold phosphate-buffered saline-azide and fixed in $1 \% \mathrm{wt} / \mathrm{vol}$ paraformaldehyde, $2 \% \mathrm{wt} / \mathrm{vol}$ glucose in phosphate-buffered salineazide. Cells were then examined within 7 days in a FACS analyser flow cytometer (Becton-Dickinson, Mountain View, Calif., USA).

The monoclonal antibody $3 G 5$ [17] was used either as reconstituted lyophilised ascitic fluid at a 1:100 dilution, or as undiluted supernatant from a growing culture of the hybridoma producing $3 \mathrm{G} 5$ (CRL 1814; American Type Culture Collection, Rockville, Md., USA). Culture supernatants from the hybridoma line P3X63Ag8, an IgG1 (kappa)-secreting variant of a non-secreting mouse myeloma line (designated X63), and from Sal 4, a hybridoma producing an IgG2b mouse anti-Salmonella antigen antibody, were used as negative control antibodies.

For identification of vascular endothelial cells, the uptake of acetylated-low density lipoprotein by these cells was employed [18]. Adherent cultures of brain or retinal pericytes, smooth muscle cells, or endothelial cells were incubated with DMEM containing fetal calf serum and $10 \mu \mathrm{g} / \mathrm{ml}$ acetylated low-density lipoprotein labelled with $1,1^{\prime}$-dioctadecyl-3,3,3',3'-tetramethylindocarbocyanine perchlorate (Dil-Ac-LDL; catalogue number BT-902; Biomedical Technologies Inc, Stoughton, Mass., USA) for $4 \mathrm{~h}$ at $37^{\circ} \mathrm{C}$ in air $/ 5 \% \mathrm{CO}_{2}$. Culture plates were then washed three times in probe-free medium and the cells were harvested using trypsin-EDTA, to produce single-cell suspensions. Cells were fixed as before, and examined by flow cytometry using a standard rhodamine filter set. Bovine vascular endothelial cells (retinal capillary and aortic) and bovine aortic smooth muscle cells were used as positive and negative control cultures, respectively.

\section{Immunocytochemistry}

During the second passage, both types of pericyte were grown on glass slide chambers (Flow Laboratories). Cells were stained for factor VIII using the Dako PAP System-20 kit (Dako Corporation, Carpinteria, Calif., USA). The primary antibody (Dako Corporation), raised against human factor VIII, had previously been shown to cross-react with bovine factor VIII [15].

\section{Growth profiles of retinal and brain pericytes}

Growth profile assays were conducted on post-confluent cultures of pericytes during the second passage. Both retinal and brain pericytes were washed two times with phosphate-buffered saline and de- 
Table 1. Characteristics of subjects from whom serum was prepared

\begin{tabular}{lcc}
\hline & Diabetic patients & Control subjects \\
\hline Race & Caucasian & Caucasian \\
Mean age (years) & $65.3 \pm 18.3$ & $64.6 \pm 14.2$ \\
Sex (male:female) & $7: 12$ & $9: 10$ \\
$\begin{array}{l}\text { Diastolic blood pressure } \\
\text { (mm Hg) }\end{array}$ & $85 \pm 15.9$ & $79.2 \pm 10.9$ \\
$\begin{array}{l}\text { Systolic blood pressure } \\
\text { (mm Hg) }\end{array}$ & $143.5 \pm 24.9$ & $142.5 \pm 29.0$ \\
$\begin{array}{l}\text { Blood glucose (mmol/1) } \\
\text { Creatinine (mmol/l) }\end{array}$ & $8.3 \pm 4.1$ & $3.4 \pm 0.9$ \\
(normal range: & & \\
$\begin{array}{l}\text { - } 06-0.12 \text { mmol/1) } \\
\text { HBA } \\
\text { (normal } \% \text { ) }\end{array}$ & $0.0819 \pm 0.02$ & $0.086 \pm 0.02$ \\
\hline
\end{tabular}

Values are given as mean $\pm \mathrm{SD}$

tached from the culture flasks using trypsin-EDTA in phosphatebuffered saline. Ten thousand pericytes of each type, suspended in $1 \mathrm{ml}$ of high glucose-DMEM supplemented with $20 \%$ fetal calf serum, were added to each of 24 wells in a 48 -well multiwell plate (Costar Corporation) on the day designated as -1 . The cultures were placed in an incubator at $37^{\circ} \mathrm{C}$ in air $/ 5 \% \mathrm{CO}_{2}$. For cell counts, the growth medium was removed from the wells, each well was washed two times with phosphate-buffered saline and a measured volume of trypsin $(0.25 \%)$-EDTA $(0.02 \%)$ was added to the well. Quadruplicate wells were counted using a haemocytometer on days $0,1,2,4,6$, and 8 for brain pericytes, and on days $0,2,4,6,8$, and 10 for retinal pericytes, since these cells grew more slowly. On days 4 and 8 , medium was removed and fresh high glucose-DMEM with $20 \%$ fetal calf serum was added to the remaining wells.

\section{Growth assay of retinal and brain pericytes cultured and assayed in high or normal glucose medium, with no reversal of glucose concentration}

Isolations of paired brain and retinal pericytes were derived from six animals. Three pairs of pericytes were grown to the second sub-culture in high glucose-DMEM supplemented with $20 \%$ fetal calf serum. For the other three pairs, pericytes were grown in normal glucose-DMEM, supplemented with $20 \%$ fetal calf serum to a final glucose concentration of $5.2 \mathrm{mmol} / \mathrm{l}$. On day -1 , post-confluent pericytes were trypsinised and $10^{4}$ pericytes, suspended in $1 \mathrm{ml}$ of medium, were plated into each of 12 wells of a 24 or 48 -well multiwell plate, using the same medium (with high or normal glucose levels) that had been used for culture. On day 0 , six wells were trypsinised and counted. These counts were designated as the initial counts. The remaining wells were washed two times with phosphatebuffered saline and $1 \mathrm{ml}$ of medium containing the appropriate level of glucose was added. Media were changed again on day 3. On day 7 , final cell counts were made from the remaining six wells.

\section{Growth assay of pericytes grown long-term in high or normal glucose medium with reversal of glucose concentration in the assay}

Retinal and brain microvessels isolated from the eyes and brains of two animals respectively, were pooled. Half of the brain microvessel material was plated into culture flasks with high glucose-DMEM supplemented with $20 \%$ fetal calf serum, and the other half with normal glucose-DMEM supplemented with $20 \%$ fetal calf serum.
Similarly, the retinal microvessel material was split into two fractions. From the material isolated, brain and retinal pericytes were grown selectively in high or normal glucose medium until the second passage. Brain pericytes grown in high or normal glucose medium were designated $\mathrm{BH}$ or $\mathrm{BN}$ respectively; retinal pericytes were similarly designated as RH or RN. An aliquot of $10^{4}$ cells of each type of pericyte in its respective growth medium was added to each well of a 48 -well multiwell plate on day -1 . On day 0 , four wells in each plate were trypsinised to provide an initial count for that plate. The remaining wells were washed two times with phosphate-buffered saline and into half of them, $1 \mathrm{ml}$ of high glucose-DMEM supplemented with $20 \%$ fetal calf serum was added and to the other half, $1 \mathrm{ml}$ of normal glucose-DMEM supplemented with $20 \%$ fetal calf serum was added. The four plates of pericytes $(\mathrm{BH}, \mathrm{BN}, \mathrm{RH}$ and $\mathrm{RN}$ ) were then incubated in air $/ 5 \% \mathrm{CO}_{2}$ at $37^{\circ} \mathrm{C}$. Since each plate of pericytes contained $50 \%$ of wells filled with either high or normal glucose medium, the pericyte type (RH, BH, RN, BN) was designated with an additional suffix, $\mathrm{H}$ or $\mathrm{N}$. Brain pericytes were counted in six replicate wells on days 2, 5 and 9; since retinal pericytes grew more slowly, counts were made on days 2,7 and 13. Media were replenished on days 3,7 and 10 . The growth of the eight types of pericytes was compared using the ratio of the final to the initial cell count. The experiment was performed on three separate occasions.

\section{Patient selection criteria}

Patients in a non-proliferative state with non-photocoagulated retinas were selected from those referred by the Diabetic Clinic of Flinders Medical Centre. Patients in renal failure were excluded. Age-matched control patients were selected from a general ophthalmology clinic. Age, blood glucose, serum creatinine, systolic and diastolic blood pressure were determined from all patients. $\mathrm{HbA}_{1 \mathrm{C}}$ levels were measured in diabetic patients. Diabetic patients were questioned about the duration of their diabetes and the type of diabetic treatment they received.

\section{Retinal status}

The fundus of 19 diabetic and 19 control patients was examined stereoscopically with the 90 dioptre indirect fundus lens, and a colour photographic survey of the diabetic patients was documented using the Airlie House Modification Schema [19]. The survey was examined subsequently to confirm the absence of proliferative retinopathy. Only control patients without retinal vascular disease were included; a post-operative fundus examination was necessary for those control patients who had extraction of a significant cataract.

\section{Patient profiles}

For the diabetic patients, the median duration of diabetes was 8 years (mean 9 years, range: 1 month - 35 years). Twelve diabetic patients were non-insulin dependent, of whom three were controlled by diet and nine with oral hypoglycaemic therapy. The remaining seven were on insulin therapy and were a mixed population of Type 1 (insulin-dependent) and Type 2 (non-insulin-dependent) diabetic patients. In the control group, 15 individuals had cataracts, two had oculoplastic lesions and two were the angiographically-normal relatives of a patient with familial exudative vitreoretinopathy. Clinical data on the subjects are shown in Table 1.

\section{Serum collection and processing}

Informed consent for venesection was given by all patients and this procedure had been approved by the Ethics Committee of Flinders Medical Centre. To allow for maximal clot retraction, $60 \mathrm{ml}$ blood 


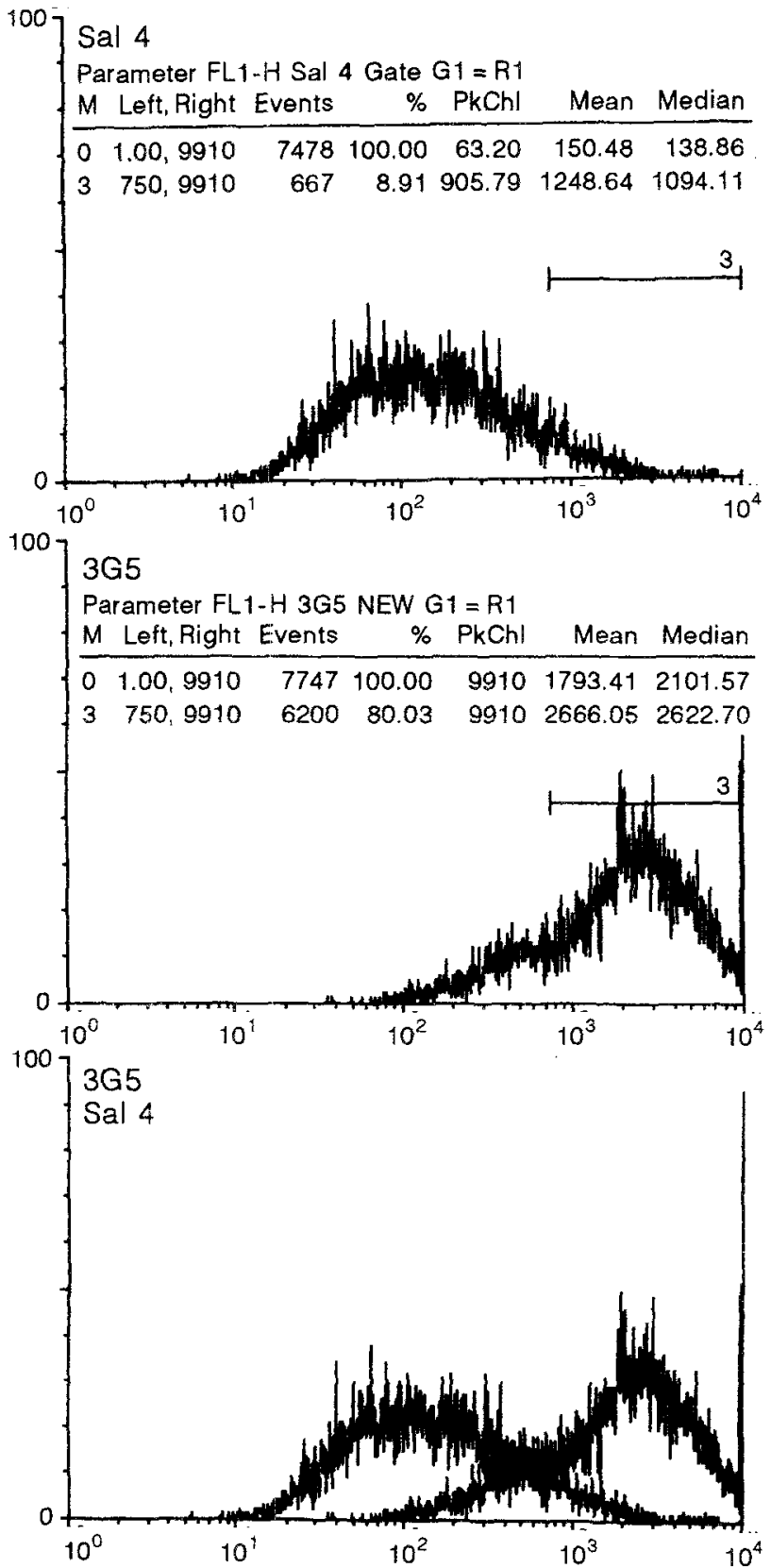

Fig.1. Representative flow cytometry profiles of trypsinised, cultured bovine brain pericytes grown in high glucose medium. Number of cells is plotted on the vertical axis and logarithm of the fluorescence intensity is plotted on the horizontal axis. The horizontal bar marks the selected gate. Approximately 5-10,000 cells were examined. Sal 4: negative control antibody; 3G5: anti-pericyte antibody; $3 \mathrm{G} 5$ and Sal 4: profiles superimposed on the same axes

samples were clotted for $4 \mathrm{~h}$ at room temperature. The resulting serum was frozen at $-70^{\circ} \mathrm{C}$. Prior to use in mitogenic assays, each serum sample was thaved at $37^{\circ} \mathrm{C}$ and half the sample was heated to $56^{\circ} \mathrm{C}$ for 30 min to inactivate complement. The other half was kept at room temperature.

\section{Mitogenic assays using human sera}

Post-confluent third passage retinal and brain pericytes from the same animal were used. To each well of a 48 -well multiwell plate, an aliquot of $10^{4}$ cells in high glucose-DMEM supplemented with $20 \%$
100

Sal 4

Parameter FL $1-H$ Sal 4 Gate G1 = R1

$M$ Left, Right Events \% PkChl Mean Median

$\begin{array}{lllllll}0 & 1.00,9910 & 8086 & 100.00 & 74.31 & 162.71 & 151.60\end{array}$

$\begin{array}{lllllll}1 & 750,9910 & 613 & 7.58 & 784.38 & 1214.33 & 1113.97\end{array}$

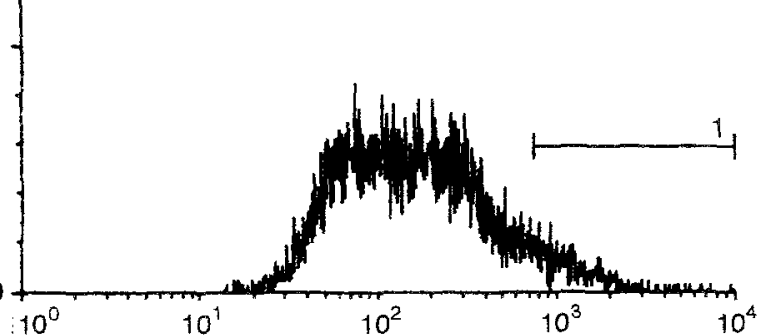

$100] 3 G 5$

Parameter FL1-H 3 G5 NEW G1 = R1

$M$ Left, Right Events \% PkChl Mean Median

\begin{tabular}{lllllll}
\hline 0 & $1.00,9910$ & 7943 & 100.00 & 2329 & 1884.25 & 2075.30
\end{tabular}

$\begin{array}{lllllll}1 & 750,9910 & 7159 & 90.12 & 2329 & 2227.99 & 2229.17\end{array}$

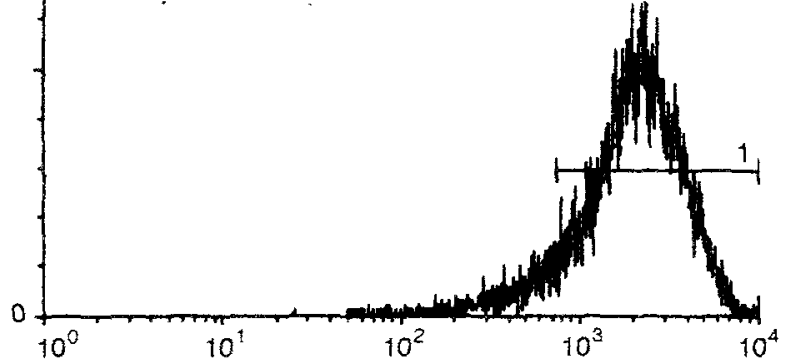

100

$3 \mathrm{G} 5$

Sal 4

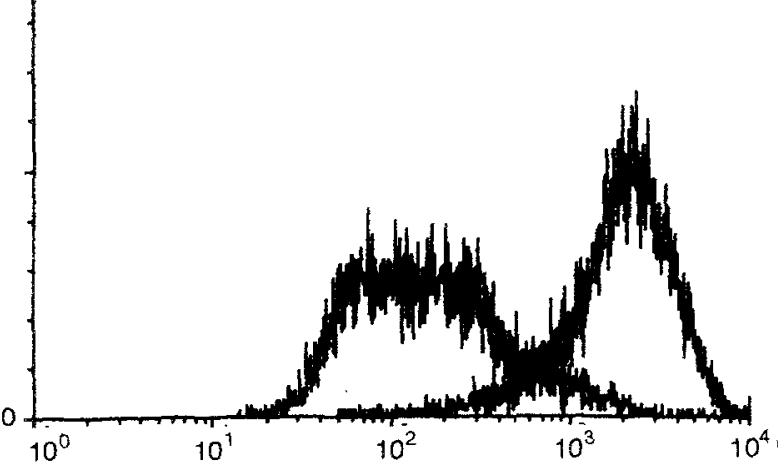

Fig.2. Representative flow cytometry profiles of trypsinised, cultured bovine retinal pericytes grown in $5.2 \mathrm{mmol}$ glucose medium. Number of cells is plotted on the vertical axis and logarithm of the fluorescence intensity is plotted on the horizontal axis. The horizontal bar marks the selected gate. Approximately 5-10,000 cells were examined. Sal 4: negative control antibody; 3G5: anti-pericyte antibody; $3 G 5$ and Sal 4: profiles superimposed on the same axes

fetal calf serum was added. At the beginning of the log phase of growth, identified by the growth profile assay during the second passage, the cells in each well were washed two times with $1 \mathrm{ml}$ phosphate-buffered saline, and $0.75 \mathrm{ml}$ of each test medium was added to the wells in quadruplicate. Four wells in each plate were trypsinised to estimate an initial cell count for that plate. After 3 days, the wells were trypsinised and a final cell count made. Each experiment was repeated two times on separate occasions.

Test media consisted of high glucose-DMEM supplemented with $20 \%$ pooled human serum from control subjects or from diabetic patients. The human serum was either heat-inactivated or non-heat-inactivated. The glucose concentrations of the high glu- 

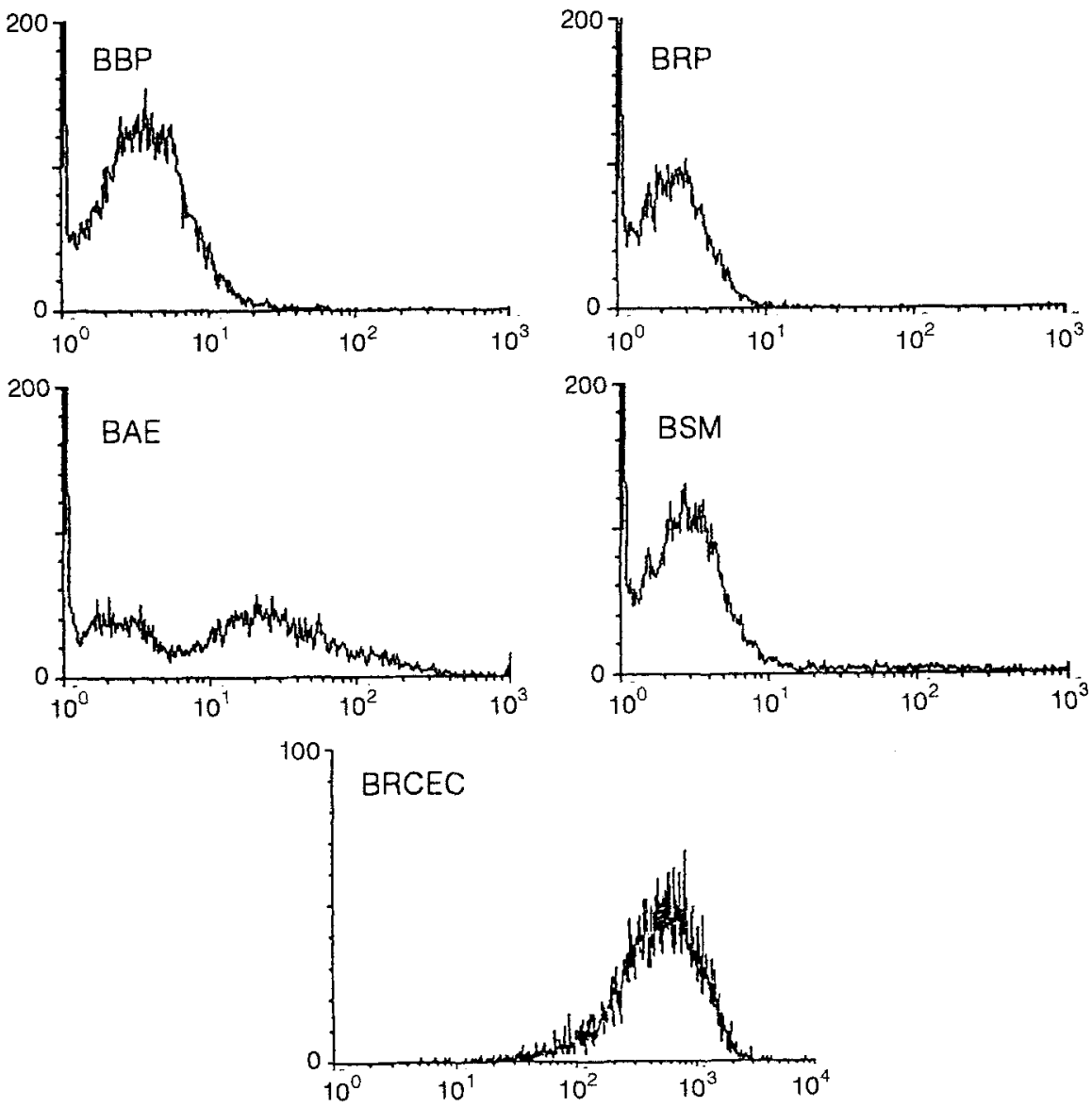

Fig. 3. Flow cytometry profiles of bovine brain pericytes (BBP), retinal pericytes (BRP), aortic endothelial cells (BAE), smooth muscle cells (BSM) and retinal capillary endothelial cells (BRCEC) following incubation with fluorochrome-labelled acetylated low density lipoprotein. Cells in the pericyte and smooth muscle cultures failed to endocytose the labelled product, in contrast to the majority of cells in the aortic endothelial and retinal capillary endothelial cell cultures

cose-DMEM supplemented with diabetic serum, and of the high glucose-DMEM supplemented with control serum, were $21.59 \pm 0.86 \mathrm{mmol} / \mathrm{l}(\mathrm{SD})$ and $20.68 \pm 0.18 \mathrm{mmol} / \mathrm{l}$, respectively (unpaired $t$-test, $p=0.0002$ ).

\section{Statistical analysis}

All statistical analyses were performed using the software package North West Analytical Statpak version 4.1 (Portland, Ore., USA).

Where growth assays were conducted in more than one multiwell plate, the growth activity of the test medium was estimated by the ratio of final to initial cell count $[15,20]$, so that differences in cell at tachment amongst multiwell plates could be eliminated. In order to compare the growth ratios, logarithms of the geometric means of the ratios (which equal the arithmetic means of the logarithms of the ratios) were used for statistical analysis. Geometric means were used since growth was measured by ratios.

For the growth assay of pericytes grown long-term in high or normal glucose medium with reversal of glucose concentration in the assay, analysis of variance and Newman-Keuls statistics were used on the arithmetic means of the logarithm of final to initial cell count, to compare the growth differences amongst the eight types of pericytes.

For the assays employing human sera and for the growth assays of retinal and brain pericytes cultured and assayed in high or normal glucose medium with no reversal of glucose concentration, the unpaired $t$-test was used on the arithmetic means of the logarithm of the ratio of the final to the initial cell counts, to compare the growth activities of the diabetic and control sera, and the proliferative rates of the brain and retinal pericytes, respectively. Linear correlations were sought between the mitogenic activities of heat and non-heat inactivated sera, and the age of diabetic and control patients, duration of diabetes, and $\mathrm{HbA}_{1 \mathrm{C}}$ levels.

\section{Results}

\section{Morphological and phenotypic analysis of pericytes}

Twelve isolations of bovine brain and retinal pericytes were made, each from an individual animal. Isolations could be cultured to the fifth passage without the morphological changes of ageing, such as cellular enlargement. Both types of pericyte stained negatively for factor VIII.

Flow cytometric analysis of unfixed, trypsinised cells indicated that approximately $85 \%$ of cells in both retinal and brain pericyte cultures grown in high glucose medium stained with the anti-pericyte monoclonal antibody, $3 \mathrm{G} 5$. Depending on the isolation, reactivity ranged from 71 $97 \%$ for retinal pericytes and from $78-93 \%$ for brain pericytes. No particular difference in reactivity was observed when pericytes were grown in normal glucose medium, as compared with high glucose medium. Representative fluorescence histograms for brain pericytes grown in high glucose medium and for retinal pericytes grown in normal glucose medium are shown in Figures 1 and 2, respectively.

The labelling of various cell populations with Dil-AcLDL, an endothelial cell marker, is shown in Figure 3. The majority of aortic endothelial cells and retinal capillary en- 


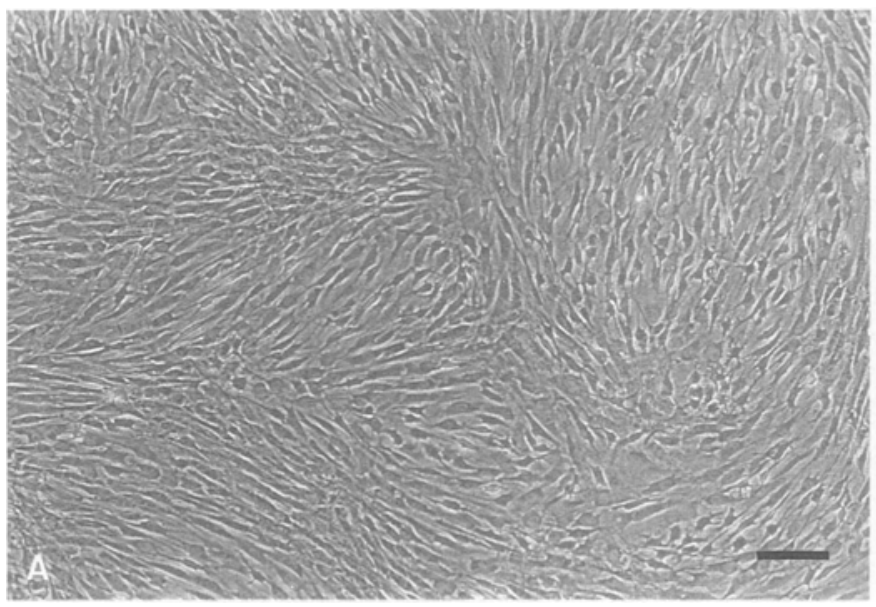

Fig. 4A,B. A Post-confluent cultures of second passage bovine brain pericytes. Note the evenly distributed and multi-layered morphology. B Post-confluent culture of second passage retinal peri-

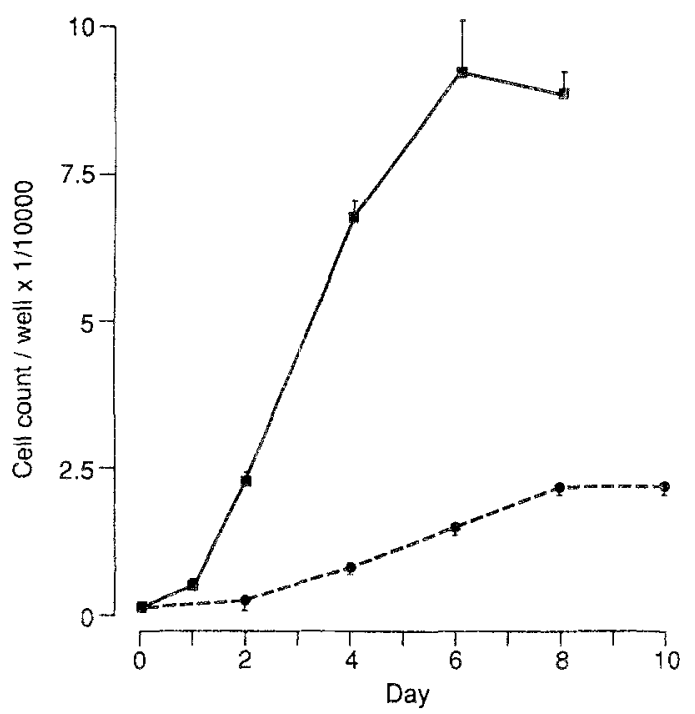

Fig.5. Growth profile of second-passage bovine retinal (--. brain (-m) pericytes in high glucose-DMEM supplemented with $20 \%$ fetal calf serum. On day $-1,10,000$ cells were seeded per $8 \mathrm{~mm}$ well. The bar denotes the SEM $(n=4)$. Brain pericyte counts were greater than retinal counts on day $2(p=0.0003)$, day $4(p=0.0004)$, day $6(p=0.0027)$, day $8(p=0.0002)$, but not on day 0 (unpaired t-test)

dothelial cells labelled with Dil-Ac-LDL (53-59\% and over $80 \%$ gated in the positive fraction, respectively), in contrast with brain pericytes ( $9 \%$ gated) and retinal pericytes ( $1 \%$ gated). Aortic smooth muscle cell populations contained $7-17 \%$ cells which labelled with Dil-Ac-LDL. These data suggest that the pericyte populations contained less than $10 \%$ contaminating capillary endothelial cells.

There was no difference in morphology between retinal and brain pericytes in the pre-confluent state. However, in the post-confluent state, brain pericytes grew in an evenly distributed multi-layered culture (Fig. 4A) whereas retinal pericytes tended to organise themselves into islands (Fig. 4B), as previously noted by Wong et al. [15]. At a very late post-confluent stage, brain pericytes did form islands and this distinction was lost.

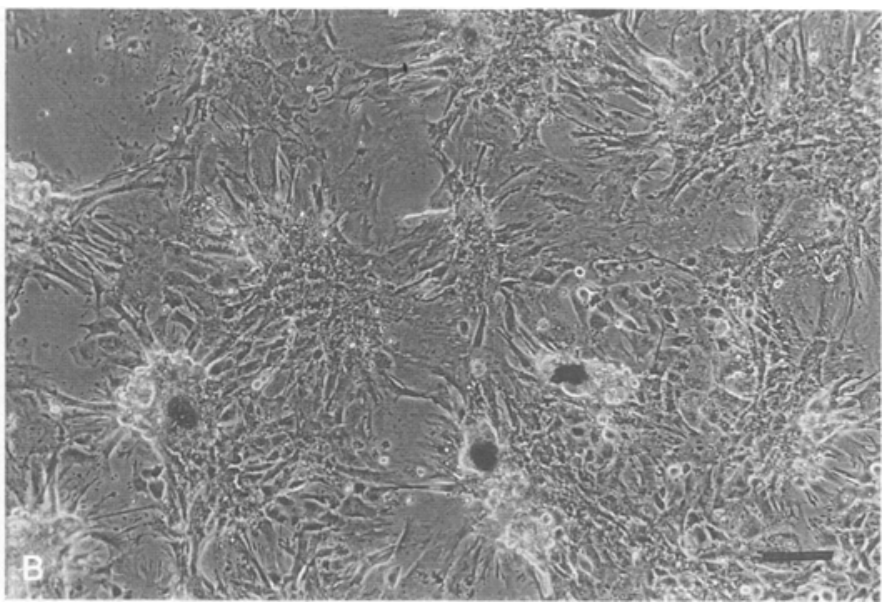

cytes from the same animal as in $\mathbf{A}$. Islands of pericytes are seen. The bar denotes $200 \mu \mathrm{m}$

Growth profile of brain and retinal pericytes in high glucose medium

Growth profiles were determined for each of four pairs of retinal and brain pericytes. The proliferative rates of brain pericytes were up to ten times greater than those of retinal pericytes in high glucose-DMEM with $20 \%$ fetal calf serum. A typical growth profile is shown in Figure 5. The cell density of brain pericytes at the end of log phase was four to five times greater than that of retinal pericytes.

Growth assay of retinal and brain pericytes cultured and assayed in high or normal glucose medium, with no reversal of glucose concentration

Six pairs of retinal and brain pericytes were used. Three pairs were cultured and assayed in high glucose-DMEM, whilst the other three pairs were cultured and assayed in normal glucose-DMEM. Brain pericytes grew significantly faster than did the retinal pairs over seven days (Fig. 6). One pair of pericytes (in normal glucose medium) grew nearly twice as fast as the other five pairs.

Growth assay of pericytes grown long-term in high or normal glucose medium, with reversal of glucose concentration in the assay

Brain and retinal pericytes grown long-term in either high or normal glucose-DMEM were transferred into normal or high glucose medium, respectively, in a reversal experiment (Fig.7). In three separate experiments monitored over a 2-week period, brain pericytes isolated, grown and assayed in either high or normal glucose medium (without reversal) grew faster than did the retinal pericytes. When the glucose concentrations were reversed during the assay, the growth ratios of the brain pericytes were still significantly greater than those of the retinal pericytes $(\mathrm{BN}-\mathrm{H}>\mathrm{RN}-\mathrm{H} ; \mathrm{BH}-\mathrm{N}>\mathrm{RH}-\mathrm{N})$, except during the middle part of the experiment when no signifi- 

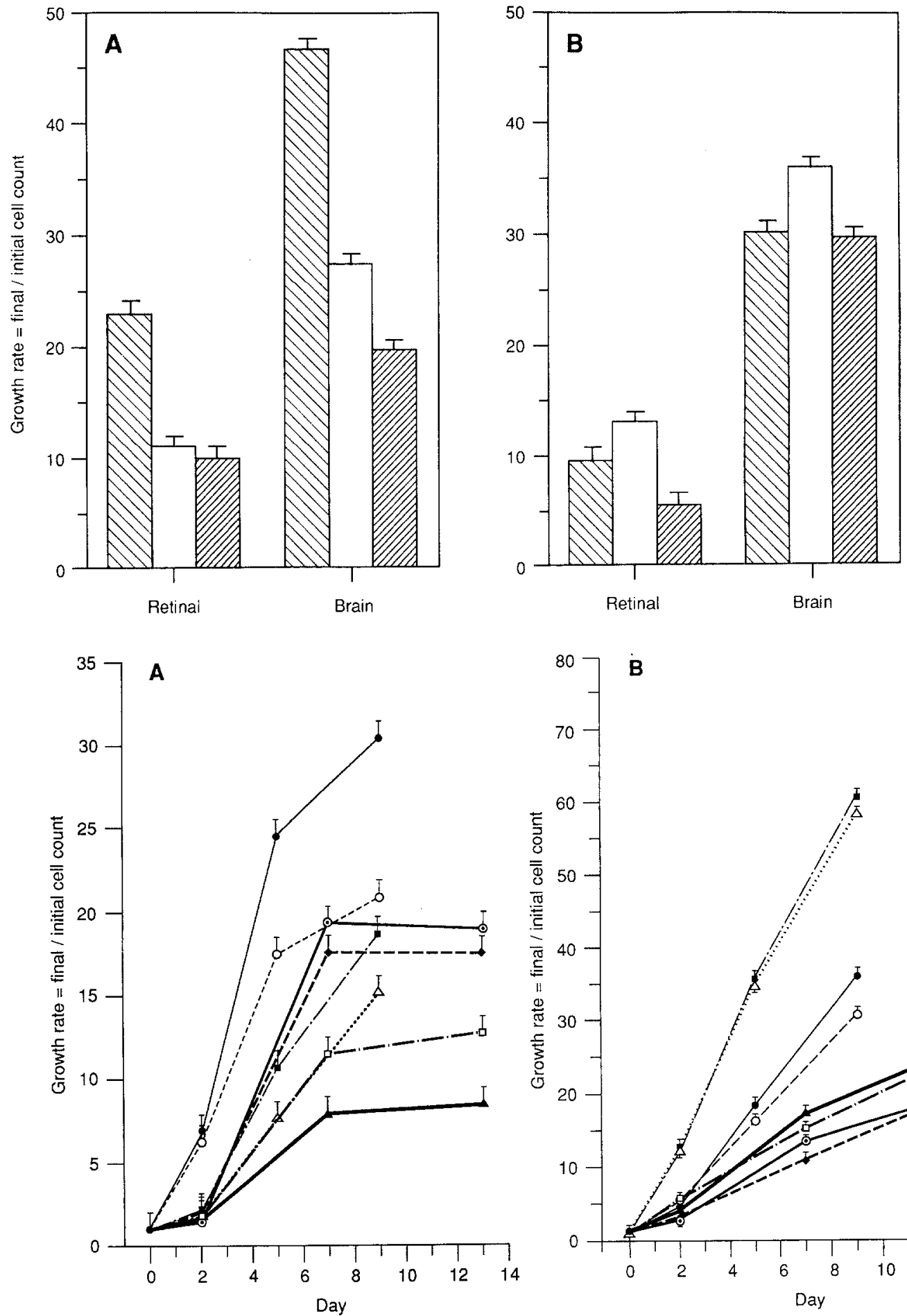

Fig. 6 A,B. Growth assay of three pairs of retinal and brain pericytes cultured and assayed in high (A) or normal (B) glucose-

DMEM supplemented with $20 \%$ fetal calf serum. The glucose concentration in high glucose-DMEM with $20 \%$ fetal calf serum was $21.4 \mathrm{mmol} / 1$, and in normal glucoseDMEM with $20 \%$ fetal calf serum was $5.8 \mathrm{mmol} / 1$. On day $-1,10,000$ pericytes cultured in either high or normal glucose to the second passage were seeded into each of 12 wells. Initial and final cells count were made on day 0 and day 7 , respectively, using six wells. The logarithm of the growth ratio of a pericyte culture in high or normal glucose medium was estimated from the logarithm of the geometric mean of the ratios of final to initial pericyte cell counts (equal to the arithmetic mean of the logarithm of the ratio). The geometric mean is the $n^{\text {th }}$ root of the multiple of $n$ data values. Pericyte pair $1 \square: 2 \square ; 3 \square$. The bar denotes $\pm \mathrm{SD}(n=6)$. For all pairs of pericytes grown in high or normal glucose medium, the growth ratios of brain pericytes were greater than those of retinal pericytes, $p \leq 0.0001$ (unpaired $t$-test).

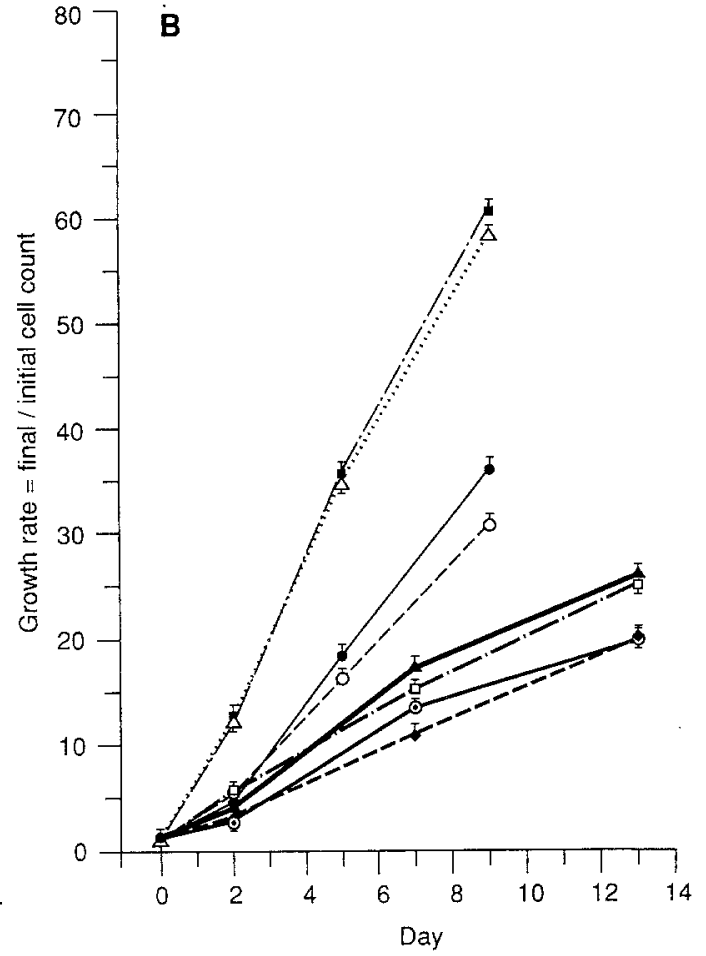

Fig. 7A, B. Growth of retinal and brain pericytes in high or normal glucose-DMEM supplemented with $20 \%$ fetal calf serum in $8 \mathrm{~mm}$ multiwell plates. $\mathrm{RH}$ and $\mathrm{BH}$ were retinal and brain pericytes respectively, grown to the second passage in high glucose-DMEM supplemented with $20 \%$ fetal calf serum. RN and BN were retinal and brain pericytes respectively, similarly grown in normal glucose-DMEM supplemented with $20 \%$ fetal calf serum. The suffix $\mathrm{H}$ or $\mathrm{N}$ after $\mathrm{RH}, \mathrm{BH}, \mathrm{RN}$ and $\mathrm{BN}$ denotes the high or normal glucose-DMEM used in the assay. Two of a total of three experiments are shown in $\mathbf{A}$ (experiment 1) and $\mathbf{B}$ (experiment 3). On day $-1,10,000$ cells were seeded per well. The bar denotes the $\operatorname{SEM}(n=6)$. $\mathrm{RH}-\mathrm{N} \square--\square, \mathrm{RH}-\mathrm{H} \rightarrow \mathbf{\wedge}$ RN-N $\odot \odot$ RN-H $\bullet \bullet$,

$\mathrm{BH}-\mathrm{N} \mathbf{\mathbf { n }}--\mathbf{\square}, \mathrm{BH}-\mathrm{H} \triangle \triangle^{--\triangle} \Delta$, $\mathrm{BN}-\mathrm{N} \bullet \mathrm{BN}-\mathrm{H} \mathrm{O}-\mathrm{O}$ cant differences were observed between some pericyte pairs.

The effect of glucose concentration on the replicative rate of pericytes was variable. In experiments one and two (Fig. 7, Table 2), the hierarchy of growth ratios at the end of the experiment was: $\mathrm{BN}-\mathrm{N}>\mathrm{BH}-\mathrm{H}$; $\mathrm{RN}-\mathrm{N}>\mathrm{RH}-\mathrm{H}$. However, in the third experiment, brain and retinal pericytes cultured long-term in high glucose medium exhibited greater growth rates than those cultured in normal glucose medium: $\mathrm{BH}-\mathrm{H}>\mathrm{BN}-\mathrm{N}$; RH-H $>$ RN-N.

\section{Mitogenic assays using human serum}

The proliferation of brain pericytes was consistently and significantly greater than that of retinal pericytes in the presence of high glucose-DMEM supplemented with human diabetic or control serum, irrespective of whether the serum had been heat-inactivated (Table 3 ) or not (Table 4). There was no significant difference in the growth-promoting activities of media containing either diabetic sera or control serum, for either brain or retinal pericytes $(p>0.05$, Tables 3 and 4$)$. Since the heat-acti- 
Table 2. Statistical analysis using one-way ANOVA and the Newman-Keuls test on the logarithms of the growth ratios in the three experiments $(1,2$ and 3$)$ shown in Figure 7

\begin{tabular}{|c|c|c|c|c|c|c|c|c|c|}
\hline \multirow[b]{2}{*}{ Experiment } & \multicolumn{3}{|c|}{ Day 2 brain vs day 2 retina } & \multicolumn{3}{|c|}{ Day 5 brain vs day 7 retina } & \multicolumn{3}{|c|}{ Day 9 brain vs day 13 retina } \\
\hline & $\overline{1}$ & 2 & 3 & 1 & 2 & 3 & 1 & 2 & 3 \\
\hline $\mathrm{BH}-\mathrm{H}>\mathrm{RH}-\mathrm{H}$ & $<0.01$ & $<0.01$ & $<0.01$ & NS & $<0.01$ & 0.01 & $=0.00$ & NS & $<0.01$ \\
\hline $\mathrm{BH}-\mathrm{N}>\mathrm{RH}-\mathrm{N}$ & $<0.01$ & $<0.01$ & $<0.01$ & NS & NS & $<0.01$ & $<0.03$ & NS & $<0.01$ \\
\hline $\mathrm{BN}-\mathrm{H}>\mathrm{RN}-\mathrm{H}$ & $<0.01$ & $<0.01$ & $<0.01$ & NS & $<0.01$ & $<0.01$ & $<0.002$ & $<0.01$ & $<0.01$ \\
\hline $\mathrm{BN}-\mathrm{N}>\mathrm{BN}-\mathrm{H}$ & NS & $<0.05$ & NS & $<0.01$ & NS & NS & $<0.01$ & $<0.01$ & NS \\
\hline $\mathrm{BH}-\mathrm{N}>\mathrm{BH}-\mathrm{H}$ & NS & NS & NS & $<0.01$ & NS & NS & $<0.01$ & NS & NS \\
\hline $\mathrm{RH}-\mathrm{N}>\mathrm{RH}-\mathrm{H}$ & \multicolumn{3}{|c|}{ Day 2 brain and day 2 retina } & \multicolumn{3}{|c|}{ Day 5 brain and day 7 retina } & \multicolumn{3}{|c|}{ Day 9 brain and day 13 retina } \\
\hline Experiment & 1 & 2 & 3 & 1 & 2 & 3 & 1 & 2 & 3 \\
\hline $\mathrm{BN}-\mathrm{N}>\mathrm{BH}-\mathrm{H}$ & $<0.01$ & $<0.01$ & - & $<0.01$ & $<0.01$ & - & $<0.01$ & $<0.01$ & - \\
\hline $\mathrm{RN}-\mathrm{N}>\mathrm{RH}-\mathrm{H}$ & NS & NS & - & $<0.01$ & $<0.01$ & - & $<0.01$ & $<0.01$ & - \\
\hline $\mathrm{BN}-\mathrm{N}<\mathrm{BH}-\mathrm{H}$ & - & - & $<0.01$ & - & - & $<0.01$ & - & - & $<0.01$ \\
\hline
\end{tabular}

NS, Not significant at the $5 \%$ level

$\mathrm{RH}$ and $\mathrm{BH}$, Retinal and brain pericytes, respectively, grown to the second passage in high glucose-DMEM supplemented with $20 \%$ fetal calf serum;
$\mathrm{RN}$ and $\mathrm{BN}$, retinal and brain pericytes, respectively, similarly grown in normal glucose-DMEM supplemented with $20 \%$ fetal calf serum vated serum was assayed at different times from the nonheat-activated serum, and on a different pair of brain and retinal pericytes, the growth activities of heat-activated serum could not be compared with non-heat-activated serum. There were no significant linear correlations between age of diabetic or control patients, duration of diabetes or $\mathrm{HbA}_{1 \mathrm{C}}$ levels, and the respective growth ratios of pericytes in any type of test medium.

\section{Discussion}

Pericyte degeneration is one of the earliest histological changes in diabetic retinopathy [4] and has been implicated in the formation of microaneurysms and new retinal vessels. More recently, in vitro findings have supported the role of pericytes in the modulation of microvascular endothelial cell growth. Bovine retinal pericytes have been shown to inhibit bovine adrenal capillary endothelial cell proliferation by contact inhibition [21]. Culture medium conditioned by bovine retinal pericytes grown in the absence of endothelial cells has been demonstrated to induce proliferation of retinal microvascular endothelial cells $[15,20]$.

We have grown enriched populations of brain and retinal pericytes from microvessels isolated from brain and retina. The majority of cells in both populations were 3G5-positive and did not incorporate low density lipoprotein. Glia may have been possible contaminants, but they seldom survive in long-term culture without prior induction of gliosis in brain or retina $[22,23]$. Since the brains and retinas used for our isolations were normal, glial contamination was likely to have been minimal.

Our mitogenic assays used the growth ratio of final to initial cell counts to compare the growth of brain and retinal pericytes in medium containing either high or normal glucose concentrations. This ratio is a reflection of the slope of the curve in the growth profile experiments and is, we believe, a better measure of growth than cell counts, since the ratio is independent of the degree of initial cell attachment.

In this study, we found that when bovine pericytes were cultured long-term in either high or normal concentrations of glucose and were then transferred into either high or normal glucose medium supplemented with fetal calf serum, the brain pericytes consistently proliferated at a greater rate than did the retinal pericytes. When pericytes

Table 3. Retinal and brain pericyte growth ratios in medium supplemented with heat-inactivated serum from 19 diabetic and 19 control patients

\begin{tabular}{llll}
\hline Serum & Pericyte & $\begin{array}{l}\text { Mean logarithm } \\
\text { of growth ratios }\end{array}$ & \pm SEM \\
\hline Diabetic patient & Brain & 0.830 & 0.041 \\
Diabetic patient & Retinal & 0.668 & 0.021 \\
Control subject & Brain & 0.819 & 0.022 \\
Control subject & Retinal & 0.656 & 0.042
\end{tabular}

Statistical comparison of growth ratios of brain and retinal pericytes in test media supplemented with heat-inactivated diabetic or control serum

Mean of $\operatorname{logs}$ of growth ratios ${ }^{\mathrm{a}}$

$p$ value (unpaired $t$-test)

Brain pericytes in diabetic serum $>$ retinal pericytes in diabetic serum

Brain pericytes in control serum > retinal pericytes in control serum

Brain pericytes in diabetic serum = brain pericytes in control serum

Retinal pericytes in diabetic serum $=$ retinal pericytes in control serum

${ }^{a}$ Growth ratio (or activity) of an individual human serum in a test medium was estimated by the logarithm of the geometric mean of the ratios of final to initial pericyte cell counts (equal to the arithmetic mean of the logarithm of the ratio). The geometric means is the $n^{\text {th }}$ root of the multiple of $n$ data values 
Table 4. Retinal and brain pericyte growth ratios ${ }^{a}$ of medium supplemented with non-heat-inactivated serum from 19 diabetic and 19 control patients

\begin{tabular}{llll}
\hline Serum & Pericyte & $\begin{array}{l}\text { Mean logarithm } \\
\text { of growth ratios }\end{array}$ & \pm SEM \\
\hline Diabetic patient & Brain & 0.855 & 0.025 \\
Diabetic patient & Retinal & 0.139 & 0.108 \\
Control subject & Brain & 0.770 & 0.110 \\
Control subject & Retinal & 0.041 & 0.110
\end{tabular}

Statistical comparison of growth ratios of brain and retinal pericytes in test media supplemented with non-heat-inactivated diabetic or control serum

Mean of logs of growth ratios

$$
\begin{aligned}
& p \text { value } \\
& \text { (unpaired } \\
& t \text {-test) }
\end{aligned}
$$

Brain pericytes in diabetic serum $>$ retinal pericytes in diabetic serum

Brain pericytes in control serum $>$ retinal pericytes in control serum

Brain pericytes in diabetic serum $=$ brain pericytes in control serum

Retinal pericytes in diabetic serum $=$ retinal pericytes in control serum

${ }^{a}$ Growth ratio (or activity) of an individual human serum in a test medium was estimated by the logarithm of the geometric mean of the ratios of final to initial pericyte cell counts (equal to the arithmetic mean of the logarithm of the ratio). The geometric mean is the $n^{\text {th }}$ root of the multiple of $n$ data values

were isolated and grown in medium containing fetal calf serum and then transferred into high glucose medium containing either diabetic or control human serum, the brain pericytes again grew more quickly than the retinal pericytes.

The reduced retinal pericyte growth rates we observed when cells grown initially in normal glucose concentrations were transferred to high concentrations of glucose were more variable than those reported by $\mathrm{Li}$ et al. [24], being apparent in only two of three experiments, and then only at the later time points tested. Variability was also observed in the growth rates of both brain and retinal pericytes grown chronically in either high or normal glucose concentrations, in that reduced growth rates were seen in high glucose concentrations in two of three experiments.

Differences in methodology may have accounted for some of this variation. Li et al. [24] synchronised the division of their pericytes by pre-treating the cells with serumdeficient medium and hydroxyurea. Our pericytes were plated and maintained in medium containing serum sufficient for growth, but without hydroxyurea. This difference, however, cannot explain the qualitative variations observed in one of our experiments, which indicates that pericytes grown long-term in high glucose concentrations may have higher replicative rates than those grown in normal glucose levels. Possibly, pericytes from individual animals show individual responses to high concentrations of glucose, in the same way that human subjects with persistently raised blood glucose levels show individual variations in their progression to diabetic retinopathy. Alternatively, different populations of pericytes may exist, with different responses to alterations in glucose concentrations. In electron microscopic studies of diabetic new vessels, pericytes have been shown to be present within the basement membrane of the vessels, suggesting the existence of a sub-population of pericytes that are able to survive and proliferate in the hyperglycaemic environment of advanced proliferative diabetic retinopathy $[25,26]$.

The mitogenic assay devised, compared both heat-inactivated and non-heated human serum, to exclude the possibility of complement-mediated destruction of pericytes in vitro. Furthermore, the assay was conducted on pericytes in such high concentrations of glucose in the test media (range 20-24 mmol/l), that any differences in the levels of glucose between diabetic and control serum supplements would have been unlikely to have altered the growth rates of pericytes significantly. We found no significant differences between the mitogenic activities of nonproliferative diabetic or control human serum for either type of pericyte. That is, we were unable to find any systemic factor(s) present in serum to cause the selective degeneration of retinal pericytes.

Although there is no specific cell marker to differentiate brain from retinal pericytes, we have consistently observed morphological differences in the organisation of early post-confluent cultures between brain and retinal pericytes from the same animal. The association of retinal pericytes into "islands" may be due to a greater affinity of these cells for other retinal pericytes, compared with the affinity that brain pericytes have for other brain pericytes, or it may be due to a decrease of anchorage-dependence to the plastic substrate. The difference in intrinsic growth rates of brain and retinal pericytes is yet another distinction between these two cell types.

In conclusion, with the qualification that these experiments were conducted with proliferating cultures of bovine, not human, cells, our findings suggest the possibility that in vascular occlusive diseases such as diabetes, the concomitant production of growth factors such as those present in serum, will lead to a greater intrinsic proliferative response in pericytes in the brain than in the retina. This response is largely independent of the environmental glucose concentration. The relative inability of the retinal pericyte to re-proliferate in response to an injury caused by diabetes may partly explain the selectivity of pericyte degeneration to the retinal circulation within the central nervous system.

Acknowledgements. This study was supported by Hoechst Australia Ltd., the Kellion Diabetic Trust, the Flinders Medical Centre. The authors thank Prof. M. Berry, Ms E. Williams, Mr. E. Johnstone, Mr. S. Standfield, Mr. I. Webster and Dr. M.Zhu for technical support, Ms W. Laffer for editorial assistance, Ms S. Rosewarne for secretarial help, Dr. R.C. Nayak (Joslin Diabetes Center, Boston, Mass., USA) for supplying the 3G5 monoclonal antibody and Dr. L. Ashman (Department of Microbiology and Immunology, University of Adelaide, Adelaide, Australia) for the gift of Sal 4.

\section{References}

1. Farquhar MG, Hartman JF (1956) Electron microscopy of cerebral capillaries. Anat Rec 124: 288-289

2. Maynard EA, Schultz RL, Pease DC (1957) Electron microscopy of the vascular bed of rat cerebral cortex. Am J Anat 100: 409443 
3. Wolter JR (1962) The pericytes of the human retina. Am J Ophthalmol 53: 981-988

4. Cogan DG, Toussaint D, Kuwabara T (1961) Retinal vascular patterns. IV.Diabetic retinopathy. Arch Ophthalmol 66:366-378

5. Speiser P, Gittelsohn AM, Patz A (1968) Studies on diabetic retinopathy. III. Influence of diabetes on intramural pericytes. Arch Ophthalmol 80:332-337

6. Kohner EM, Henkind P (1970) Correlation of fluorescein angiogram and retinal digest in diabetic retinopathy. Am J Ophthalmol 69: 403-414

7. Tilton RG, Faller AM, Burkhardt JK, Hoffmann PL, Kilo C, Williamson JR (1985) Pericyte degeneration and acellular capillaries are increased in the feet of human diabetic patients. Diabetologia 28: 895-900

8. Addison DJ, Garner A, Ashton N (1970) Degeneration of intramural pericytes in diabetic retinopathy. Br Med J 1: 264-266

9. Ashton N, Tripathi R (1977) The problem of selective pericyte injury. 9th European Conf Microcirculation, Antwerp, Bibl Anat No 16. Karger, Basel, pp 16-23

10. Frank RN (1991) On the pathogenesis of diabetic retinopathy. A 1990 update. Ophthalmology 98: 586-593

11. Kennedy A, Frank RN, Varma SD (1983) Aldose reductase activity in retinal and cerebral microvessels and cultured vascular cells. Invest Ophthalmol Vis Sci $24: 1250-1258$

12. Akagi Y, Kador PF, Kuwubara T, Kinoshita SH (1983) Aldose reductase localization in human retinal mural cells. Invest Ophthalmol Vis Sci 24: 1516-1519

13. Kador PF, Akagi T, Terubayashi $H$, Wyman M, Kinoshita JH (1988) Prevention of pericyte ghost formation in retinal capillaries of galactose-fed dogs by aldose reductase inhibitors. Arch Ophthalmol 106: 1099-1102

14. Engerman RL, Kern TS (1990) Effect of sorbinil on retinopathy in diabetic dogs and galactosemic dogs. Invest Ophthalmol Vis Sci 30 [Suppl 4]: 124 (Abstract)

15. Wong HC, Boulton ME, Marshall J, Clark P (1987) Growth of retinal capillary endothelia using pericyte conditioned medium. Invest Ophthalmol Vis Sci $28: 1767-1775$

16. Zola H (1987) Monoclonal antibodies: a manual of techniques. CRC Press, Boca Raton, pp 9-103

17. Nayak RC, Berman AB, George KL, Eisenbarth GS, King GL (1988) A monoclonal antibody (3G5) - defined ganglioside antigen is expressed on the cell surface of microvascular pericytes. J Exp Med 167: 1003-1015

18. Voyta JC, Via DP, Butterfield CE, Zetter BR (1984) Identification and isolation of endothelial cells based on their increased uptake of acetylated-low density lipoprotein. J Cell Biol 99: 2034-2040

19. Diabetic retinopathy study research group (1981) Report number 7 - a modification of the Airlie House classification of diabetic retinopathy. Invest Ophthalmol Vis Sci 21:210-226

20. Wong HC, Boulton M, McLeod D, Bayly M, Clark P, Marshall J (1988) Retinal pigment epithelial cells in culture produce retinal vascular mitogens. Arch Ophthalmol 106: 1439-1443

21. Orlidge A, D'Amore PA (1987) Inhibition of capillary endothelial cell growth by pericytes and smooth muscle cells. J Cell Biol 105: 1455-1462

22. Lindsay RM, Barber PC, Sherwood MR, Zimmer J, Raisman G (1982) Astrocyte cultures from adult rat brain. Derivation, characterization and neurotrophic properties of pure astroglial cells from corpus callosum. Brain Res 243: 329-343

23. Sarthy PV (1985) Establishment of Muller cell cultures from adult rat retina. Brain Res 337: 138-141

24. Li W, Shen S, Khatami M, Rockey JH (1984) Stimulation of retinal capillary pericyte protein and collagen synthesis in culture by high-glucose concentration. Diabetes 33: 785-789

25. Tanaguchi Y (1976) Ultrastructure of newly formed blood vessels in diabetic retinopathy. Japan J Ophthalmol 20: 19-31

26. Wallow IHL, Geldner PS (1980) Endothelial fenestrae in proliferative diabetic retinopathy. Invest Ophthalmol Vis Sci 19: 11761183

Received: 3 January 1992

and in revised form: 26 May 1992

Dr. H.C. Wong

Department of Ophthalmology

Flinders Medical Centre

Bedford Park SA 5042

Australia 